\title{
Theory and Application Progress of Long Bone Segmental Defect Reparation and Reconstruction
}

\author{
Rui Jiang ${ }^{1}$, Ziyan Zhang ${ }^{2}$, Han $\mathrm{Wu}^{1}$, and Chao Zhang ${ }^{3}$ \\ ${ }^{1}$ Department of orthopedics, China-Japan Union Hospital of Jilin University, Jilin 130033, China \\ ${ }^{2}$ Department of orthopedics, The Second Hospital of Jilin University, Jilin 130041, China \\ ${ }^{3}$ Department of ophthalmology, The Second Hospital of Jilin University, Jilin 130041, China \\ Corresponding Author: Chao Zhang: zhangchao0701@163.com
}

Keywords: long bone, segmental defect, repetitive research

Abstract: Objective: To summarize domestic and foreign research on reparation and reconstruction progress for long bone segmental defects in recent years. Method: The first author applied computers to retrieve articles of PubMed database, Chinese Journal Full-text Database and VIP Database about long bone segmental defect reparation and reconstruction according to English search term; bone defect, long bone reconstruction, tissue engineering of bone, scaffolds, bone reconstruction, bone graft, bone tumor; tumor resection, musculoskeletal tumors, regeneration, autografts' and Chinese search term 'large segment, bone defect, bone tumor, soft tissue tumor, enbolc resetion, tissue engineering and bone graft' from January 1990 to December 2012. A total of 104 related articles were searched, and 52 related articles were included for further study. Results and conclusion: Traditional repair methods of long bone segmental defects include autogenous bone graft, allogeneic bone graft, bone lengthening, artificial prosthetic replacement, etc. Development of prosthesis suitable for children limb salvage demands and consistent with children growth characteristics becomes a challenge for children limb salvage. People develop prosthesis capable of constant lengthening in order to solve the problem, which is used in limb salvage reconstruction for children bone tumor, with progress of medical technology. Various artificial bones, especially gradually application of bone tissue engineering and gene treatment gene treatment in bone defect repair, will further improve the remediation effect of bone defects. It is future research direction.

\section{Introduction}

Large segmentbone defect refers to long bone defect caused by congenital osteopathy, trauma, infection, bone tumor resection and other causes, wherein the length is longer than threshold value-1.5 times of long bone diameter or long bone defect is larger than 1/5-1/4 of the bone[1]. Some scholars have successfully used it in animal models [2]. The bone defects lower than the threshold value can heal themselves, and bone defects higher than threshold value cannot heal themselves. The repair and reconstruction after long bone segmental defect has always been a hot spot and difficulty in orthopedics. Autogenous or allogeneic bone graft, artificial material substitute placement, artificial prosthetic replacement, bone handling, bone lengthening and other method 
have been applied in clinic, but they all have their own limitations, thereby limiting wide application thereof. The development of the microsurgical technique provides new vitality for bone defect reparation and reconstruction, new direction is proposed, it is still defective for treatment of long bone segmental defect, which is not widely applied clinically. Large segment bone defect treatment by tissue engineering technology has been a hot topic in the current research by the development of tissue engineering and gene transfer technology makes, and it has achieved initial results [3-6].

\section{Data and Methods}

Data source: Computers were applied for retrieving related articles of PubMed database (http://www.ncbi.nlm.nih.gov/pubmed?myncbishare=smulib), Chinese Journal Full-text Database (http://www.cnki . net/) and VIP Database(http://lib.cqvip.com/) according to English search term: 'bone defect, long bone reconstruction, tissue engineering of bone, scaffolds, bone reconstruction, bone graft, bone tumor; tumor resection, musculoskeletal tumors, regeneration, autografts' and Chinese search term 'large segment bone defect, repair and reconstruction' from January 1990 to December 2012. Original works, overview, review, case report, meta-analysis, etc. were retrieved and studied. Microsurgery and In Enneking WF (ed): LimbSalvage in Musculoskeletal Oncolog were artificially retrieved, and a total of more than 30 Chinese and English articles were retrieved.

Inclusion criteria (1) contents mentioned in the article should include research on reparation and reconstruction clinical experiments of long section bone defects or report in the aspects of long section bone defect reparation and reconstruction curative effect and complications; (2) recently published articles or articles published on authoritative journals were selected in the same field.

Exclusion criteria: repetitive research

Data extraction: 104 articles were obtained through initial review by computers, titles and abstracts were read for early screening, thereby eliminating repeated reports in both Chinese and English literature, repeatedly reported cases with different observation control contents, factors and purposes as well as contents in the literature unrelated to reparation and reconstruction of long section inclusion criteria.

Quality assessment: 52 articles consistent with inclusion criteria included clinical effect observation of trauma-caused long segment bone defect, therapeutic effect evaluation of tumor-caused long segment bone defect, clinical experiment research on animal model, etc.

\section{Results}

\subsection{Autogenous bone graft}

Autogenous bone has the best functions of bone conduction, bone induction and bone formation, which is the first-choice bone supply source for defect repair [7]. Because it has the advantages of fast healing, no rejection reaction, etc., it is an ideal bone defect reparation and reconstruction method [8]. Commonly used bones for autogenous bone graft include ilium, fibula and ribs, but supply bones are limited with poor bone strength, which is not suitable for reconstruction of load bearing bone defects [9]. Therefore, it is limited during application in lower limb bone defect reconstruction. However, the upper limb does not bear load, the influence of reduction on upper limb function is low. Therefore, it is more suitable for applying autogenous bone graft repair [10]. When large segmentbone defect is repaired, intramembrane or entochondrostosis can be discovered on the surface of grafted bone, the central zone is completely absorbed due to ischemic necrosis, holes or fibers are formed, it is difficult to shape the bone and restore the mechanical stability of limbs quickly. Bone defect healing is changed into fracture healing due to development of 
microsurgical techniques, especially the clinical application of autogenous bone graft with vascular pedicles, thereby promoting the therapeutic effect of bone defect repair.

\subsubsection{Autogenous bone graft of anastomotic vessels:}

Autogenous bone graft of anastomotic vessels is an effective therapeutic method [11-12] for the defect of limb long bone large segment, which can not be easily repaired by traditional bone grafts. Currently, it is believed that fibula graft with blood supply is the most effective method for repairing segmental long bone defects [13]. However, fibular nutrient vascular pedicle is located deeply, free transplantation trauma is larger, some patients suffer from congenital vascular malformation and anatomical variations or calf blood vessel damage caused by trauma, thereby increasing operation difficulty and risk (14-15). How to reduce the risk of surgery, improve the safety and accuracy of surgery, and reduce the dependence of the operator on the past experience becomes the "bottleneck" for the further promotion and application of the operation mode. 9 patients of long segment bone defect with requirements on fibular free graft repair include 3 cases with humerus defect ( 2 cases with humerus pulmonary bone defect and 1 case with post-operative recurrence after aneurysmal bone cyst), respectively 1 case with radioulna oboslete bone defect, 2 cases with femurbone defect (including 1 case with combined chronic osteomyelitis) and 2 cases with tibia bone defect. The defect length is 6 to $18 \mathrm{~cm}$ with an average of $9.6 \mathrm{~cm}$, wherein 3 cases are combined with local soft tissue defect. Tools software is utilized for reconstructing patient digital fibula flap and blood vessels three-dimensionally according to patient preoperative spiral CT two-dimensional images, thereby engaging in preoperative design and simulation operation accordingly, and guiding cutting of intra-operative fibula flap. Free fibula flap transplantation [16] is adopted. Postoperative recovery rate of limb function is $90.4 \%$ on average, wherein femur defect functions are less recovered, which is followed by tibia defect. Imaging evaluation: bone healing is initiated 3 to 8 months after surgery with an average healing time of 4.5 months. The transplanted fibula is gradually thickened in a compensated mode due to stress effect after bone healing. It is thickened similar to the recipient site skeleton 10-30 months after surgery. There are no further fracture and bone resorption, only 1 case had mild angulation. All patients have no donor site ankle joint arthrodesis and abnormal sense in peroneal nerve control site. The ankle joint activity is normal.

\subsubsection{Autogenous free bone graft of bone marrow}

Since marrow is rich in BMP, and other bone growth factor and osteoblasts, the monocyte, blood platelet, etc. can generate growth factor, therefore autogenous red marrow not only has own ossification in bone defect repair, but also can induce and promote change of other tissue cells in the fracture into osteoblasts. Therefore, autogenous free bone graft with autogenous red marrow significantly improves local osteogenesis ability. Anterior superior spine or posterior superior iliac spine can be selected in the supply area. Li Bo, etc. [17] adopt small allogeneic bone to fully mix with red marrow of autogenous ilium, which is grafted on both ends of large allogeneic bone and fixed with steel plate or intramedullary needle and hollow forcing screws, the grafted large allogeneic bone and autogenous bone surface are gradually integrated and vascularized for 6 months to 2 years. The bone density of bone block is gradually reduced to the level the same as autogenous bone. Bone graft layer and autogenous bone bed gap is fuzzy, which is penetrated by bone trabecula, the bone density of grafted bone and surrounding bone bed tend to be consistent, and bones are integrated with vascularization performance. Qiu Yuanqiang [18] applies static interlocking intramedullary nail combined to autogenous free bone graft with autogenous red marrow for treating large segment bone defect. Obvious porosis is discovered 1 month after grating, and the bone defect is healed well 6 months later. 


\subsection{Allogeneic bone graft}

Since allogeneic bone has normal bone strength, the bone can be selected and utilized according to shape and size of the defect area, the sources are wide, etc. It has been widely used in reparation and reconstruction therapy of long bone segmental defect.The allogeneic bone is mostly combined with autogenous bone or autogenous red marrow for transplantation jointly in order to improve the ossification of allogeneic bones [17]. It is jointly applied with artificial joint prosthesis in order to improve the mechanical stability after tumor enbolc resetion [19]. Moran, etc. [20] applies the method of inserting fibula into allogeneic marrow cavity freely to repair 7 child patients with bone tumors, the postoperative mean follow-up period is 36 months, the limb salvage rate is $100 \%$, the average bone healing time is nine months with no allogeneic bone fracture and infection. with the development of neoadjuvant chemotherapy and the improvement of assembled artificial joint technology, limb salvage surgery has become the main method for the bone neoplasm therapy. In bone tumor limb salvage surgery, because quite a number of bone tumors invade for more than $1 / 2$ of the whole bone length, soft tissue and bone defects are larger after enbolc resetion, tumor artificial joint prosthesis is equipped complexly, the fixing arm in the host bone is short, there are insufficient important muscle and ligament attachment points, the post-operative load-bearing requirements of lower limb one tumor are high, the patients are prone to artificial joint fracture, dislocation, loosening, etc. in the early stage. Conventional prosthesis can not provide satisfactory fixation effect [19]. The artificial joint and the host bone are stabilized by three fixation modes, including mechanical fixation, biological fixation and composite fixation. Research shows that good mechanical fixation can achieve effective mechanical stability, which is conducive to the postoperative functional exercise in the early stage and biological healing between allogeneic bone and host bone, the fixing arm of artificial joint prosthesis in the host bone is lengthened, thereby obtaining satisfactory biological fixation, improving the healing effect of artificial joint, and prolonging the service life [23-24]. Since some scholars successfully apply allogenic bone and artificial prosthesis for bone repair respectively, allogenic bone transplantation and artificial prosthetic replacement are still two most effective methods for reparation and reconstruction of large segmentbone defect $[25,26]$ at present. A large number of allogenic bone s have been clinically applied with the progress of various hypothermia, cell, immunity and sterilized antigen removal technology, thereby bringing hope to patients with various large segmentbone defects. Some scholars adopt allogeneic frozen bone grafts, external fixing bracket is combined to support and repair large segmentbone defects. It has the advantages of deep cryogenic treatment of the allogenic bone s, low immunogenicity and excellent biological mechanics performance, which is advantageous to healing of grafted bone. The external supporting structure maintains stable environment for early healing of bone defects, mechanical stimulation is provided for accelerating bone defect healing later [27]. allogenic bone transplantation with the blood vessels has received much attention with the progress of transplantation immunology, since the late 20th century [28]. allogenic bone transplantation with blood vessels has unique advantages of keeping its own natural structure, shape, bone induction ability, etc. After the bone is grafted successfully, it will become a permanent tissue of the receptor, and its application value is worth of expectation. However, current allogeneic bone graft with blood vessel is still in the experimental research stage, Its clinical application is relatively lagging behind the other tissues and organs for prolonging the patient's life, and higher requirements are also proposed. The progress of current allogenic bone transplantation with blood vessel is combined to make breakthrough in the following aspects: (1) solution of the bone source; (2) improvement of bone preservation technique; (3) tissue matching technique suitable for allogenic bone transplantation with blood vessel; (4) short-term use of safe, low toxicity, effective and economical immune inhibitor; (5) ideal immune tolerance induction plan. It 
is expected that allogenic bone transplantation with blood vessels has wide prospect in the aspect of repairing large segmentbone defect [29]. There are many factors affecting large segment allogeneic bone healing and alternation. The method of increasing large segment allogeneic bone surface area, and combining with BMP and other bone growth factors are adopted with broad application prospects in order to promote large segment allogeneic bone healing and replacement, reduce fracture, no healing, bone graft absorption, joint collapse and other complications. [20]

\subsection{Bone transportation and bone lengthening}

Basic method of bone lengthening: firstly, the skeleton on both ends of the long bone are fixed with an external fixation support; secondly, bone cortex is truncated on the distal end of the bone defect (the cancellous bone in the marrow cavity of the backbone site is not truncated), the soft tissues around the bone defect should be reserved as far as possible, the osteotomy end is gradually guided and opened 5-7 days later. The osteotomy sites are connected through callus proliferation. Meanwhile, both cut ends of the bone defect are close to each other mutually until they contact mutually, or healing is promoted through local grafts, or the bones can be healed by themselves. The efficacy is certain during treatment of large segment bone defect. Compared with other methods, such method has irreplaceable advantage to some extent [31]. It can also be used for treating limb bone defect diseases caused by trauma and tumor, congenital malformation, bone infection, etc. [32].

\subsection{Applicable of extensible prosthesis}

Limb salvage therapy is mostly adopted as the clinical therapy of teenager limb malignant bone tumors, which is mainly based on artificial prosthetic replacement after extensive tumor resection. Many problems can be caused after joint replacement for children with immature skeletons, such as different lengths of limbs, pelvis tilt, spine lateral bending, etc. Development of prosthesis suitable for children limb salvage demand and consistent with growth developmental characteristics of children is a challenge topic. People developed an extensible prosthesis in order to solve the problem, which is used for limb salvage reconstruction for children bone tumors. Such extensible prosthesis has the advantage that the prosthesis can be extended regularly and artificially with children growth. The ill limb and healthy limb can grow synchronously, etc. [33] The extensible artificial prosthesis (Stanmore I) was developed in 1976, which has been developed into the fourth generation at present- biological extensible prosthesis (Fitbone prosthesis). Clinical application report shows that Fitbone prosthesis has relatively few postoperative complications, and the length of limbs can reach up to $60 \mathrm{~mm}$ (average $40 \mathrm{~mm}$ ). In 2002, Wang, etc. respectively carried out extensible artificial bone joint prosthetic replacement successfully for three children suffering from tibia upper segment, femur lower segment and upper segment malignant bone tumors, who are 10 to 12 years old. The therapy plan is precedent in China at present. All 22 children suffering from malignant bone tumors from 6 and 13 years old, reported by scholars, completed successful limb salvage through applying extensible prosthesis limb salvage operation. The operation time was two to three hours, and the intraoperative bleeding was 300-500 mL. 8 children had not undergone extensible surgery because such cases include 6 cases with distant metastasis or death, 1 case with peripheral infection of prosthesis, and 1 case with current limb reduction of less than $2 \mathrm{~cm}$ [34]. 14 patients underwent 34 extension surgeries with average extension of $1(0.6-1.5) \mathrm{cm}$ each time. The longest follow-up time was 86 months, the the patient has been extended for 6 times and a total of $6 \mathrm{~cm}$. The mid-stage follow-up shows that the functions are consistent with expectation of bone tumor doctors after affected limb prosthesis extension. Currently, children bone tumor prosthesis extension is gradually changed from medium surgery part replacement into minimally invasive 
extension or even noninvasive extension [35], which provides good opportunity for further development of limb salvage operation in children.

\subsection{Artificial bone substitute materials}

It is urgent to study various artificial bone materials in order to overcome the inherent shortcomings of autogenous bone or allogeneic bone. The ideal artificial bone material should have the following characteristics: good biocompatibility; bioabsorptivity; bone conductivity; bone induction and reliable bone strength; the structure is similar to bone, which is cost-effective and easy to operate, etc.. However, there is no material that can meet all above requirements. Therefore, research on ideal artificial bone graft substitute materials is still the key and hot spot in future study. The graft of composite materials is the combination of bone conduction matrix, bone induction and osteogenic active substances such as growth factor, marrow tissue, etc..

Bone cement and cortical bone joint transplantation is applied for treating limb malignant bone tumorenbolc resetion and then the bone is reconstructed. Such method is convenient with significant curative effect in the aspects of bone healing and prevention of early complications. Hydroxyapatite ceramics, fibroblast growth factor 2 and blood vessel bundle joint transplantation are applied for prefabricating vascularization artificial bone, which is used for reparation and reconstruction of rabbit radial bone defect. It is better than single artificial bone restorative materials in the aspects of bone inductivity and osteogenesis performance. However, all substitute materials are applied in the body as foreign materials, and they have the risks of aseptic inflammation, etc. Current artificial bone materials commonly used in clinical practice include various biological ceramics, bone cement, tissue artificial bone, etc. Biological ceramics mainly include hydroxyapatite, biological activity glass, aluminum oxide single crystal biological ceramic, etc. They have good biological compatibility, excellent bone conduction and bone induction effect, higher mechanical strength, corrosion resistance and chemical stability. It has advantage in the aspect of treating bone defect. Bone cement materials mainly include calcium phosphate bone cement, carrying recombinant BMP 2/poly lactic-co-glycolic acid SPSPM/ calcium phosphate bone cement composites, acrylics bone cement, etc. It has the advantages of wide sources, easy tissue preparation, convenient use, easy shaping, low heat release during curing, self-gardening property, etc. with certain development prospects, but it also has the risk of patient allergic reaction.

\subsection{Tissue engineering bone}

Foreign scholars, etc. [33] implanted calf periosteal cells in multilayer woven polyglycolic acid non-woven mesh stent, then it was transplanted in nude mice in vivo, finally it was confirmed that it can be proliferated as skeleton. Some scholars comprehensively put forward the concept, method, present situation and prospect of bone tissue engineering research. Bone tissue engineering refers that the separated autogenous high concentration osteoblasts and marrow stromal stem cells or chondrocytes [32] are planted in a natural or synthetic cell scaffold or extracellular matrix with good biocompatibility after in vitro cultivation and amplification, which can be degraded and absorbed by human body gradually. The biomaterial scaffold can provide cells with survival three-dimensional space, which is beneficial for cells to get enough nutrients, exchange gas and eliminate waste, cells can grow on a three-dimensional scaffold in a precast form. Then, the cell hybrid materials can be implanted on bone defect sites. The planted bone cells are proliferated during gradual degradation of biological materials so as to achieve the purpose of repairing bone defect. The method can solve defects of various bone defect repair methods, and it becomes a brand-new treatment mode engineering bone for bone defect reparation and reconstruction, which is applied in bone defect repair. Satisfactory clinical results have been achieved. Vascularization and 
bone regeneration are the two most basic links in the process of bone healing. However, because the vascularization of the tissue engineering bone is a difficulty in clinical study, it is still in the experimental stage at present, which can not be widely used in clinical practice [36]. In recent years, new and effective means are provided for the repair of bone defect on the one hand, better clinical treatment effect is achieved on the other hand due to rapid development of bone tissue engineering. Meanwhile, principles and methods of bone tissue applied engineering and life science belong to a multi-disciplinary field. Some high-tech technologies, such as nanotechnology, have been applied to the development of scaffold materials. The ideal tissue engineering bone should be approximate to autogenous grafted bone: (1) the compatibility is excellent, graft versus host reaction and graft rejective reaction are not generated in the implant; (2) Self-proliferation and osteogenesis are achieved, and it can secrete extracellular matrix for local deposit calcification. (3).It induces osteogenesis, which contains cytokines promoting the growth of new bone and is acted on local bone defect through slow release method, thereby promoting vascularization and osteoblasts proliferation and differentiation. (4) The method has bone conduction, the graft material can be used as a scaffold make host cells and blood vessels into the material for forming new bone. Then, the graft material is absorbed and gradually replaced by new bones. The three basic biological factors of bone tissue engineering include the follows: seed cells, scaffold materials and osteogenic factors. Therefore, it is very important to make rational use of cytokines, reasonably construct scaffold materials, and regulate appropriate expression of osteoblasts during construction of tissue engineering bone. Jian Yuekui, etc. [37] studied and confirmed that bone defects can be repaired successfully through histological compound artificial bone constructed by marrow stromal cell combined bioactive glass. In addition, osteoblasts and endothelial cells are jointly inoculated in nano-hydroxyapatite collagen composite materials to construct nanostructures.

\section{Acknowledgments}

This work was supported by the study of c-Myc and mTOR inhibitors in the combined targeting therapy of osteosarcoma. Jilin provincial finance department. (No 3D517P373430)

\section{References}

[1] Christopher JB, Marc AA, Ruth SH. Iliac crest bone graft harvest donor site morbidity. A statistical evaluation. Spine, 1995, 20(9):1055-1060.

[2] Younger EM, Chapman MW. Morbidity at bone graft donor sites. JOrthop Trauma, 1989, 3(3):192-195.

[3] Zhou Xiao, Sheng Xiaowu, Zeng Yong. Research progress and problems of bone tissue engineering. Tissue Engineering and Reconstruction Surgery, 2016, 12(5):319-321.

[4] Tan Xinyu, Ren Zhijian, Tan Duxun. Experience of applying allogeneic cortical bone segment graft in treating limb bone ununion. China Orthopedics Clinical and Basic Research Magazine, 2009, 1(1):66-67.

[5] Venkatramani H, Sabapathy SR, Dheenadayalan J, et al. Recon-struction of post-traumatic long segment bone defects of the lowerend of the femur by free vascularized fibula combined with allograft (modified Capanna's technique). Eur J Trauma Emerg Surg, 2015, 41(1):17-24.

[6] Zheng Hua, He Shengjiang, Wang Qunbo. Treatment of old age humerus proximal fracture with locking steel plate plus allogenic bone transplantation. Journal of Traumatic Surgery, 2012, 14(2):130-132.

[7] Yu Shaobin, Dong Qirong, Wang Yabin. Application of allogenic bone transplantation in shift intraarticular calcaneal fracture operation. China Reparation and Reconstruction Surgery, 2008, 22(7):869-872.

[8] He Bo, Huang Bo, Jiang Shuxiang, etc. Stage II reconstruction and reparation femur open fracture accompanied by bone defect with large segment allogenic bone transplantation. Orthopedic Journal of China, 2010, 18(16):1386-1388.

[9] Vulcano E, Murena L, Cherubino P, et al. Treatment of severe post-traumatic bone defects with autologous stem cells loaded on al-logeneic scaffolds. Surg Technol Int, 2012(22):291-301.

[10] Enneking WF. A system of staging musculoskeletal neoplasms. Instr Course Lect, 1988(37):3-10.

[11] Capanna R, Bufalini C, Campanacci M. A new technique for re-constructions of large metadiaphyseal bone defects, a combined graft allograft shel plus vascularized fibula. Orthop Traumatol Surg Res, 1993, 24(3):159-177. 
[12] Abed YY, Beltrami G, Campanacci DA, et al. Biological recon-struction after resection of bone tumours around the knee: long-term follow-up. J Bone Joint Surg, 2009, 91(10):1366-1372.

[13] Huang Nanxiang, Tu Chongqi, Duan Hong, etc.. Clinical analysis on limb salvage treatment of 55 cases suffering from limb osteosarcoma with frozen large segment allogenic bone transplantation. West China Medical Journal, 2007, 22(1):91-92.

[14] Enneking WF, Campanacci DA. Retrieved human allografts: a clin-icopathological study. J Bone Joint Surg Am, 2001, 83(7):971-986.

[15] Reikeras $O$, Shegarfi H, Naper C, et al. Impact of MHC mismatch and freezing on bone graft incorporation: an experimental study in rates. J Orthop Res, 2008, 26(7):925-931.

[16] Zhang Dawei, Li Zhenhui, Pei Guoxian. Immunology comparison of compound cyclosporin allogeneic bone and and frozen allogeneic bone graft. Pharm J Chin PLA ; Pharmaceutical Jo, 2014, 35 (3):266-269.

[17] Huang Yongjun, Huang Dong, Zhang Dawei, etc.. Repair of bone defect effect by FTY-720P through inhibiting osteoclast and forming enhanced allogeneic bone. China reparation and reconstruction Surgery, 2016, 30(4):426-431.

[18] Sims L, Kulyk P, Woo A. Intraoperative culture positive allograt bone and subsequent postoperative infections: a retrospective re-view. Can J Surg, 2017, 60(2):8016.

[19] Zhang Xiaowen, Wang Wei. Analysis on 21 cases of allogenic bone transplantation postoperative infection. Chin J Prim Med Pharm, 2002, 9(9):838.

[20] Yasuda H, Yano K, Wakitani S, et al. Repair of critical long bone defects using frozen bone allografts coated with an rhBMP-2-retai-ning paste. J Orthop Sci, 2012, 17(3):299-307.

[21] M ankinHJ, Doppett SH, Sullian TR, et a.l Ostearticular and in-tercalary allograft transplantation in the management of malig-nant tumor ofbone. Cancer, 1982, 50:613 -630.

[22] Zu Zhonghe and Cai Weishan, Guo Qifeng. Treatment of femurlarge segmentbone defect by combined fibula graft with monitoring flap . Chinese Microsurgery, 2000, 23:29-31.

[23] Guo Qifeng, Xu Zhonghe, Wen Shifeng. Treatment of femurlarge segmentbone defect by combined fibula graft with monitoring flap; Chinese Microsurgery, 2003, 26:299-300.

[24] Zhang Guangming, Yang Yunfa, Xu Zhonghe, et al. Repair of large segment bone joint defect after bone tumor resection with composite bone graft; Chinese Microsurgery, 2003, 26:18, 1-183.

[25] Amini AR, Laurencin CT, Nukavarapu SP. Differential analysis of peripheral blood and bone marrow derived endothelial progenitor cells for enhanced vascularization in bone tissue engineering. J Orthop Res, 2012, 9:1507 $-1515$.

[26] Harris CT, Cooper LF. Comparison of bone graft matrices for human mesenchymal stem cell-directed osteogenesis. J Biomed Mater Res A, 2004, 4:747 -755.

[27] Fedorovich NE, Haverslag RT, Dhert WJ, et al. The role of endothelial progenitor cells in prevascularized bone tissue engineering:development of heterogeneous constructs. Tissue Eng Part A, 2010, 7:2355 -2367.

[28] Yuan Mengjiang, Wang Ya, Yao Yao, etc. Influence of periosteum on own free grafted bone revascularization; International Journal of Stomatology, 2010, 3:257 -261.

[29] Wang Jindong, Wang Yan, Liang Bingsheng. Related research on bone graft vascularization and bone reconstruction; Journal of Practical Orthopaedics, 2006, 2:130-133.

[30] Lei L, Kanglai T, Liu Y, et al. Study of biological safety of scaffold material with heterogeneous deproteinized bone. Chin J Traumatol, 2006, 4:234 -237.

[31] Brey EM, Cheng MH, Allori A, et al. Comparison of guided bone formation from periosteum and muscle fascia. Plast Reconstr Surg, 2007, 4:1216-1222.

[32] Carter S, Braem K, Lories RJ. The role of bone morphogenetic proteins in ankylosing spondylitis. Ther Adv Musculoskelet Dis, 2012, 4:293 -299.

[33] Heliotis M, Lavery KM, Ripamonti U, et al. Transformation of a prefabricated hydroxyapatite/osteogenic protein-1 implant into a vascularised pedicled bone flap in the human chest. Int J Oral Maxillofac Surg, 2006, 3:265 -269.

[34] Jeon O, Song SJ, Kang SW, et al. Enhancement of ectopic bone formation by bone morphogenetic protein-2 released from a heparin-conjugated poly ( L-lactic-co-glycolic acid) scaffold. Biomaterials, 2007, 17:2763 -2771.

[35] Seebach C, Henrich D, Kahling C, et al. Endothelial progenitor cells and mesenchymal stem cells seeded onto beta-TCP granules enhance early vascularization and bone healing in a critical-sized bone defect in rats. Tissue Eng Part A, 2010, 6:1961-1970.

[36] Lin Mingfeng, Song Jianrong. Research status and progress of allogenic bone transplantation of anastomotic vessels. Medical Review, 2007, 6:42, 3-425;

[37] Jian Yuekui, Li Qihong, Liu Lei, et al. Vascularization observation on the repair of large segment bone defects by heterogeneous deprotein-bone complex. Orthopedic Journal of China, 2007, 10:78-770. 